\title{
Biopsia de endocérvix
}

\author{
Edwin Alberto Hoyos Usta*
}

\begin{abstract}
RESUMEN: Se realizó un estudio comparativo de la calidad de las muestras de patología y los diagnósticos definitivos histopatológicos expedidos por un mismo patólogo, de los tejidos obtenidos mediante raspado endocervical con cureta de Novak y mediante escisión cuboidal con radiofrecuencia en $\mathbf{5 6 2}$ pacientes estudiadas por sospecha citológica de neoplasia intracervical. Se evidencia la inadecuada calidad del material producto del raspado, contrario a la excelente muestra de la radiofrecuencia, que influye favorablemente en un diagnóstico certero.
\end{abstract}

PALABRAS CLAVES: Biopsia endocervical, radiofrecuencia, raspado endocervical.

SUMMARY: A comparative study was done of the quality of pathological samples and the last histopathologic diagnostics given by the same Pathologist, between the samples obtained through endocervical curetage with tire Novak cureta and through the cuboidal excision with radiofrecuence, in $\mathbf{5 6 2}$ patients having abnormal citology compatible with intraepitelial neoplatia.

KEY WORDS: Endocervical sample, radiofrecuence, endocervical curetage.

\section{Introducción}

La calidad del tejido endocervical para estudio histopatológico obtenido tradicionalmente mediante Cureta de Novak, ha sido durante mucho tiempo motivo de preocupación de los ginecólogos que manejan a diario la patología cervical ya que vemos frecuentemente informes de patología en donde el tejido es considerado inadecuado para estudio o inadecuado para dar un diagnóstico inequívoco.

Tratando de establecer cuán seguros y confiables son los resultados histopatológicos expedidos por los patólogos en la unidad de patología cervical con respecto al raspado endocervical, hemos adelantado un estudio retrospectivo para comparar este procedimiento con muestras de tejido endocervical obtenidas mediante una sonda de radiocirugía, representado por una porción de tejido sólido de forma cuboidal, correspondiente a la porción distal del canal endocervical, con una altura que oscila entre los cinco y ocho milímetros y lados de cuatro $\mathrm{mm}$ y cinco $\mathrm{mm}$.

\section{Material y métodos}

Se revisaron 562 casos clínicos de pacientes entre los 20 y 60 años que fueron atendidas en la consulta de la Unidad de Patología Cervical de La Liga Contra El Cáncer Seccional Bogotá durante los años de 1990 a 1992 por presentar citología sospechosa o positiva para Neoplasia Intraepitelial Cervical, de las cuales 466 pacientes fueron sometidas a raspado endocervical (R.E.C) con Cureta de Novak, subdividiéndolas en dos subgrupos dependiendo de si la Colposcopia fue satisfactoria o no.

\footnotetext{
* Ginecólogo-Oncólogo.
}

La Colposcopia fue considerada satisfactoria cuando se visualizó completamente la unión escamocolumnar y/o se delimitó claramente el límite de las lesiones sospechosas de Neoplasia Intraepitelial Cervical (NIC), resultando 239 casos con Colposcopia insatisfactoria y 227 con Colposcopia satisfactoria.

A un segundo grupo de 96 pacientes con citología sospechosa para NIC y con colposcopia insatisfactoria se les obtuvo una muestra del tejido del canal endocervical con una sonda de radiocirugía obteniendo una muestra cuboidal de más o menos $8 \mathrm{~mm}$ de alto por $5 \mathrm{~mm}$ de ancho que contiene perfectamente conservada la arquitectura glandular endocervical y una buena cantidad de tejido del estroma que lo sostiene. (Esquema 1)

Las características microscópicas de las muestras correspondientes a todo el universo de las pacientes estudiadas, fue analizada directamente del protocolo de patología individual y clasificados en siete grupos así:

- Material insuficiente para estudio cuando el contenido de la muestra estaba constituido por moco, sangre, endometrio ítsmico o por células glandulares sueltas.

- Material glandular endocervical cuando microscópicamente se observaron fragmentos de glándulas endocervicales.

- Endocervicitis.

- Neoplasia Intraepitelial Cervical.

- Carcinoma Escamocelular.

- Cambios celulares por papiloma virus.

- Pólipo Endocervical.

En cada uno de los grupos de pacientes ya establecidos (grupo 1: pacientes con colposcopia satisfactoria y raspado endocervical, grupo 2: pacientes con colposcopia satisfactoria y raspado endocervical, grupo 3: pacientes con colposcopia insatisfactoria y biopsia endocervical con radiocirugía) se investigó y analizó la frecuencia con 


\section{Esquema 1 \\ REPRESENTACION ESQUEMATICA DE LA \\ MUESTRA CUBOIDAL OBTENIDA CON \\ RADIOFRECUENCIA DEL CANAL ENDOCERVICAL. \\ SE RESALTA LA INTEGRIDAD DE LA \\ ARQUITECTURA DEL CANAL}

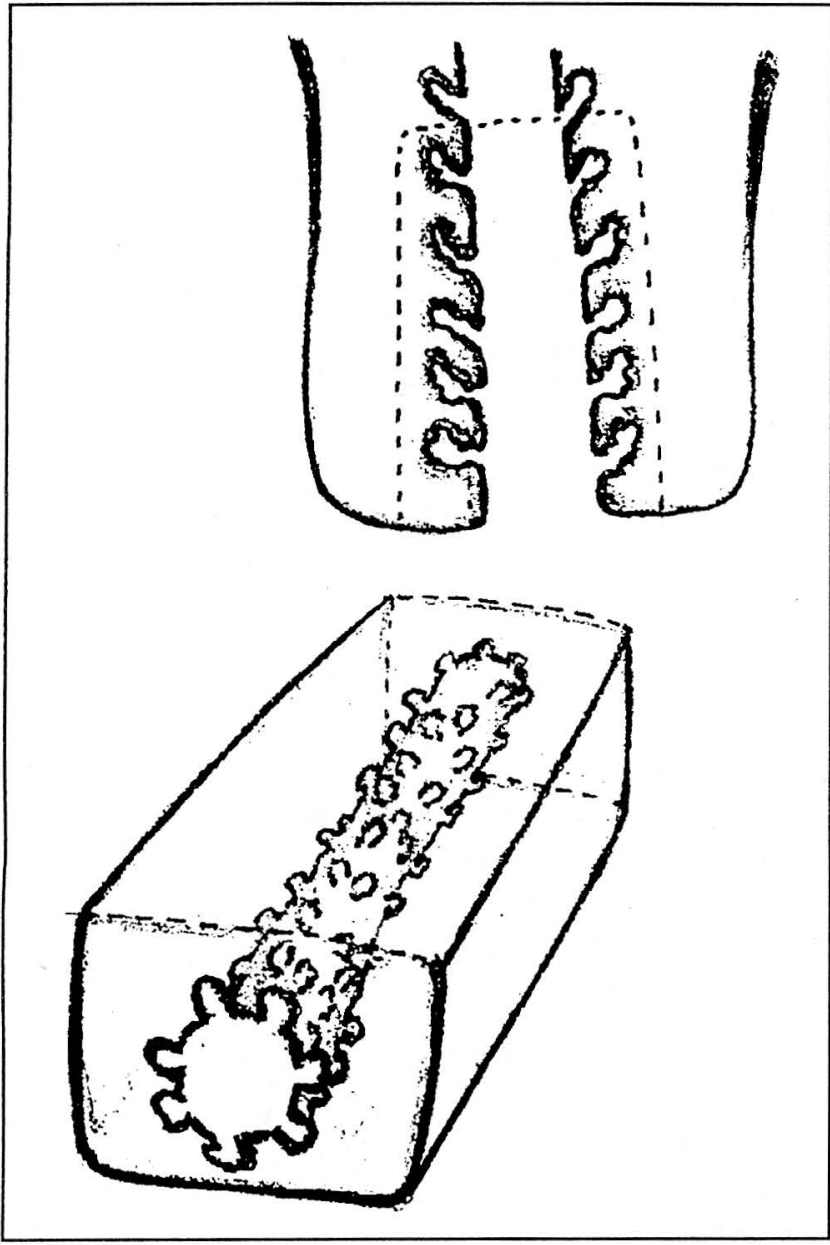

que se presentaron los diferentes diagnósticos microscópicos anotados. Se compararon los hallazgos microscópicos correspondientes a NIC en los dos grupos de pacientes con colposcopia insatisfactoria, para confrontar el raspado endocervical con la biopsia endocervical con radiocirugía. Además en este último grupo se analizó la relación entre la sospecha citológica para el grado de severidad de la neoplasia con el diagnóstico histopatológico de la muestra.

\section{Hallazgos}

De las 239 pacientes con colposcopia insatisfactoria y raspado endocervical encontramos los siguientes diagnósticos histopatológicos: (Figuras 1 y 2)

- Muestra insuficiente para diagnóstico: 39.

Endocervicitis y glándulas endocervicales: 85.

- Neoplasia Intraepitelial Cervical: 103.

- Carcinomas: 10.

Cambios por Papiloma Virus Humano: 2.
Figura 1

C.1 Dx HISTOPATOLOGICOS DE 239 PACIENTES CON COLPOSCOPIA INSATISFACTORIA $Y$ RASPADO ENDOCERVICAL

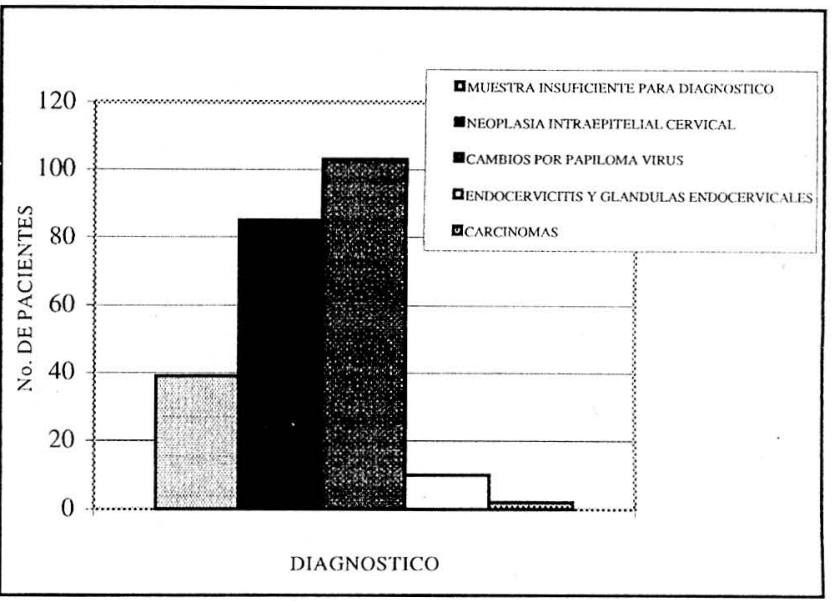

Figura 2

C.2 PORCENTAJE DE Dx DE PACIENTES CON COLPOSCOPIA INSATISFACTORIA Y RASPADO ENDOCERVICAL

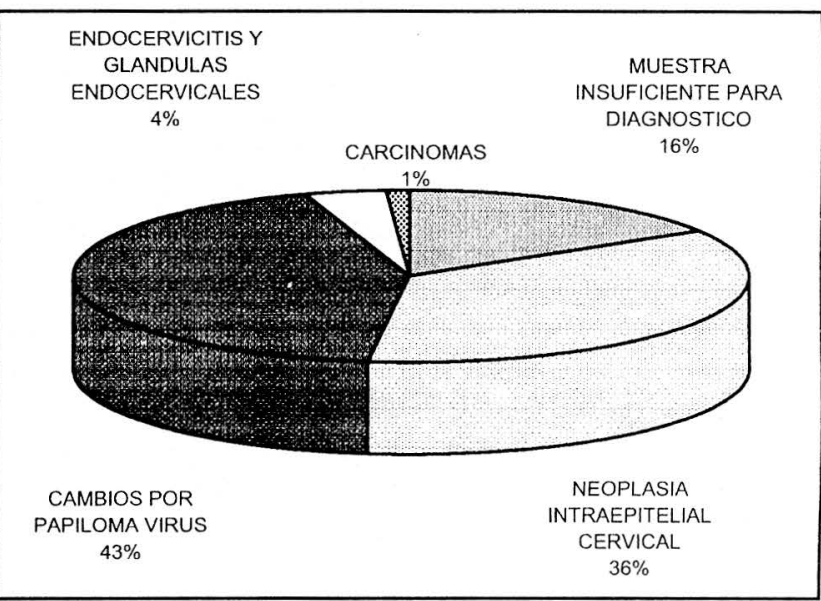

En el segundo subgrupo de 227 pacientes con colposcopia satisfactoria y raspado endocervical, fueron encontrados los siguientes diagnósticos histopatológicos: (Figuras 3 y 4 )

- Muestra insuficiente para diagnóstico: 58.

- Endocervicitis y glándulas endocervicales: 158.

- Neoplasia Intraepitelial Cervical: 11.

- Carcinomas: 0.

- Cambios por Papiloma Virus Humano: 0.

En el grupo de 96 pacientes sometidas a biopsia con radiocirugía, 95 muestras fueron diagnosticadas como Neoplasia Intraepitelial Cervical, un carcinoma infiltrante y ninguna muestra fue considerada insuficiente para diagnóstico. (Figuras 5 y 6 ). 
Figura 3

C.3 Dx HISTOPATOLOGICOS DE 227 PACIENTES CON COLPOSCOPIA SATISFACTORIA Y RASPADO ENDOCERVICAL

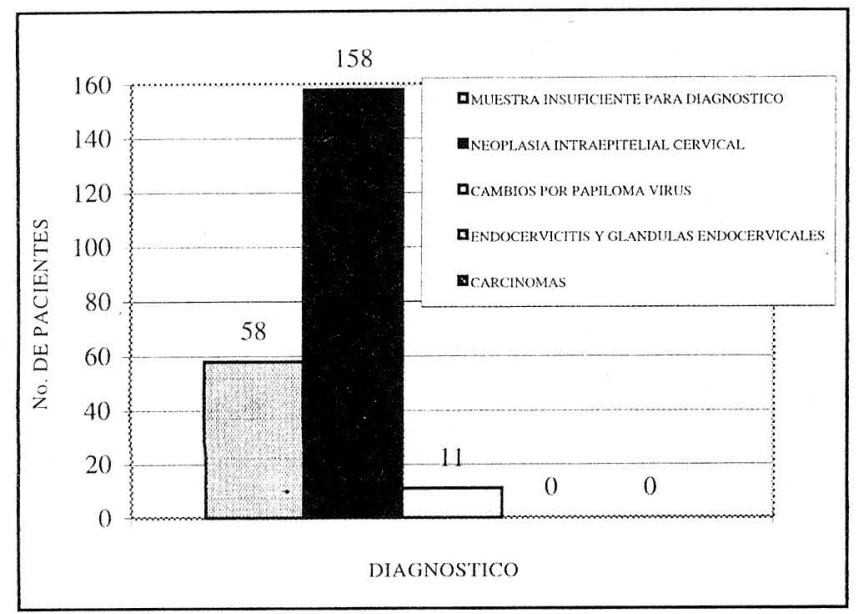

Figura 4

C. 4 PORCENTAJE DE DX DE PACIENTES CON COLPOSCOPIA SATISFACTORIA Y RASPADO ENDOCERVICAL

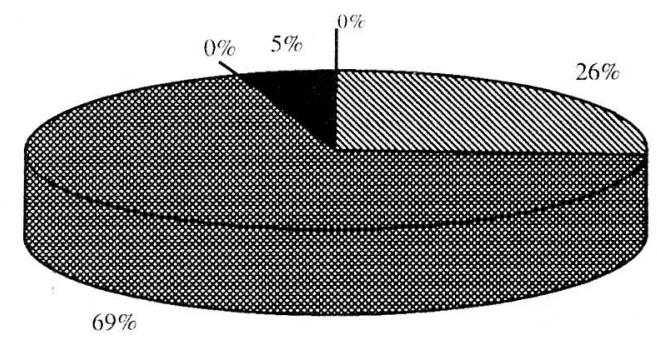

\$MUESTRA INSUFICIENTE PARA DIAGNOSTICO QENDOCERVICITIS Y GLANDUL $i$ ENDOSERVICALES ? DNEOPLASIA INTRAEPITELIAL CERVICAL DCARCINOMAS

CAMBIOS POR PAPILOMA VIRUS

El grado de severidad de las 95 Neoplasias Intraepiteliales se presentó con la siguiente frecuencia: 46 NIC grado I, 26 NIC grado II y 23 NIC grado III.

Al comparar la sospecha citológica en cuanto a la severidad de la neoplasia, encontramos 20 citologías sospechosas para NIC I cuyos diagnósticos histológicos definitivos fueron 19 NIC grado I, 8 NIC grado II y 3 NIC grado III. 28 citologías sospechosas de NIC grado II cuyos diagnósticos histológicos definitivos fueron 7 NIC grado I, 13 NIC grado II y 8 NIC grado III. 22 citologías sospechosas de NIC grado III cuyos diagnósticos histológicos fueron 4 NIC grado I, 5 NIC grado II, 12 NIC grado III y 1 carcinoma infiltrante.

En 16 pacientes la citología fue reportada como grado II con atipias severas o sospechosa o grado III sin clasificar el tipo de neoplasia al que podría corresponder, encontrándose en el estudio histopatológico 14 neoplasias intraepiteliales grado I y 2 neoplasias intraepiteliales grado II.
Figura 5

\section{C.5 Dx DE 96 PACIENTES SOMETIDAS A BIOPSIA CON RADIOCIRUGIA}

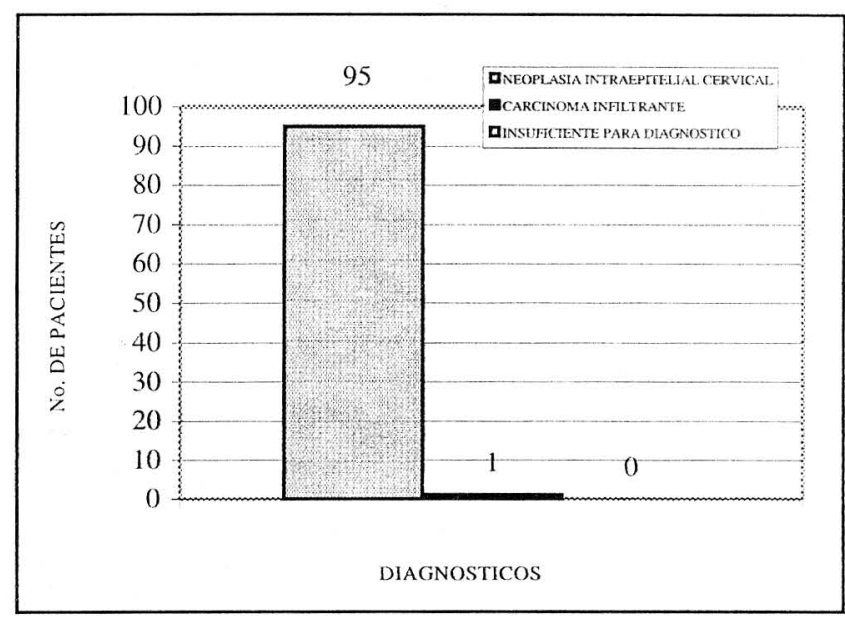

Figura 6

C.6 PORCENTAJE DE DX DE PACIENTES SOMETIDAS A BIOPSIA CON RADIOCIRUGIA

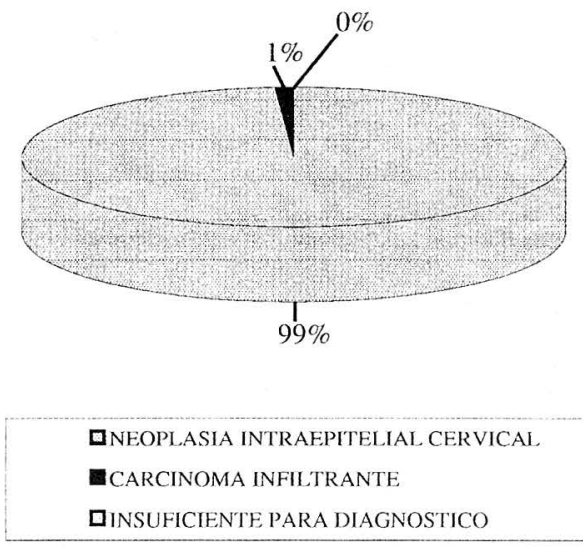

\section{Discusión}

Es evidente la imprecisión del estudio realizado con el tejido obtenido mediante Raspado Endocervical con Cureta de Novak y el alto riesgo que esto conlleva al dejar a muchas pacientes expuestas a la progresión silenciosa de una neoplasia endocervical no diagnosticada a tiempo. Tal grado de falencia tiene una lógica explicación si razonablemente analizamos el procedimiento utilizado: El contacto de la Cureta de Novak en el interior del Canal Endocervical, con su movimiento de adentro-afuera provocando el arrancamiento traumático del tejido glandular superficial a la luz, evidentemente no suministrará tejido que conserve la estructura glandular que incluya estroma y criptas glandulares, sitio este de localización frecuente de neoplasias. Así, el material obtenido y enviado a patología consistirá en una mezcla de moco, sangre y escasos fragmentos glandulares sin ningún tipo de orientación para el patólogo. Este tejido es preparado en bloque y estudiado en su totalidad apare- 
Lámina 1

FOTOGRAFIA COLPOSCOPICA DE UNA PIEZA CUBOIDAL ENDOCERVICAL OBTENIDA CON RADIOCIRUGIA. 2. LECHO CERVICAL POSTERIOR AL PROCEDIMIENTO

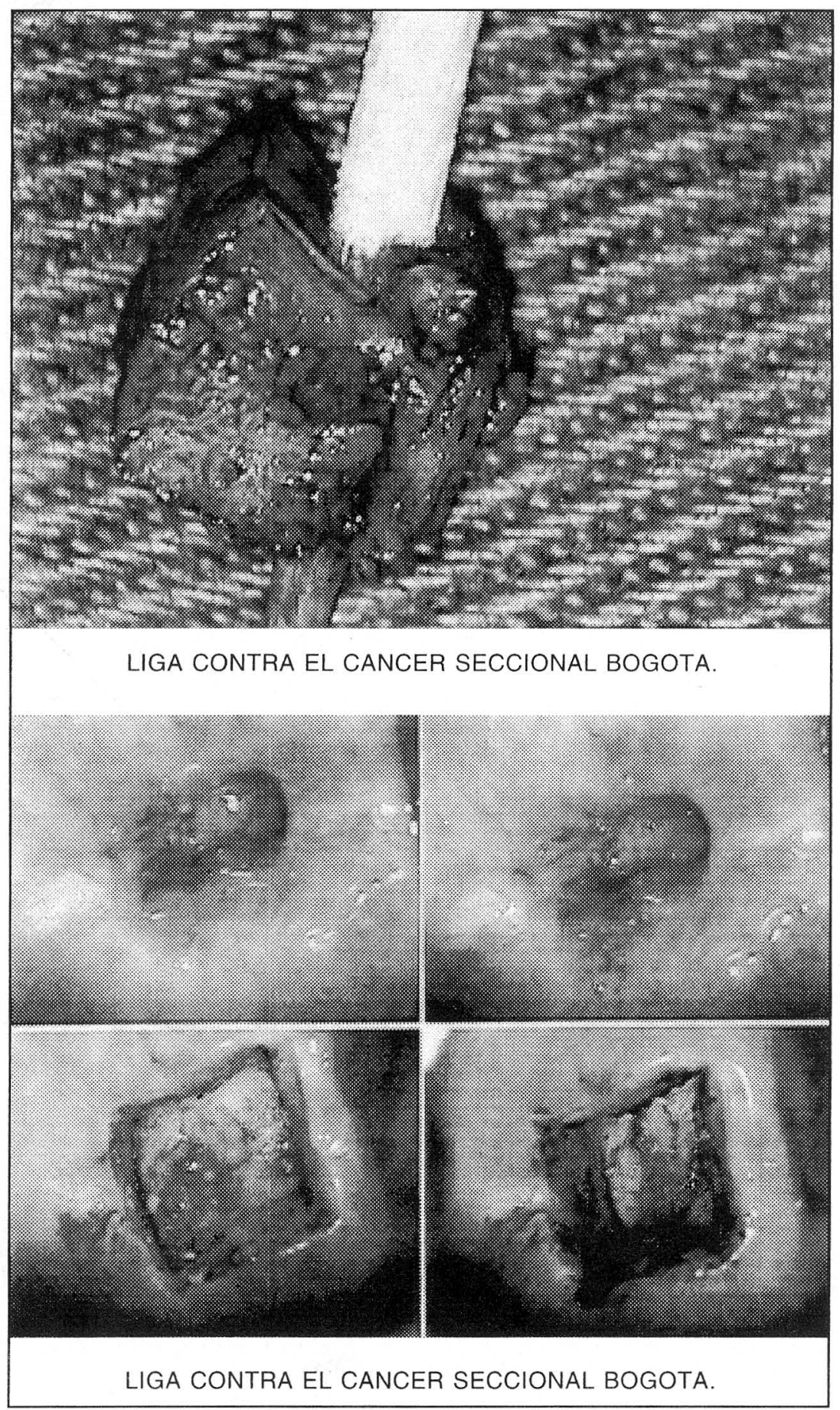

ciendo microscópicamente gran cantidad de eritrocitos esparcidos entre los que se podrá localizar fragmento glandulares constituidos por pocas células y generalmente sin estroma de sostén (Esquema 2).

En algunas ocasiones, el ginecólogo inadvertidamente puede sobrepasar el orificio cervical interno en el momento de realizar el R.E.C. y obtener tejido glandular de endometrio ítsmico, prestándose esta situación a que el patólogo incurra en un error diagnóstico por la seudoestratificación nuclear característicos del endometrio.

Sólo en casos excepcionales de Poliposis Endocervicál es posible obtener mediante R.E.C. tejido adecuado para 
Esquema 2

REPRESENTACION ESQUEMATICA DEL RASPADO ENDOCERVICAL CON CURETA DE NOVAK. LA MUESTRA ES OBTENIDA POR ARRANCAMIENTO

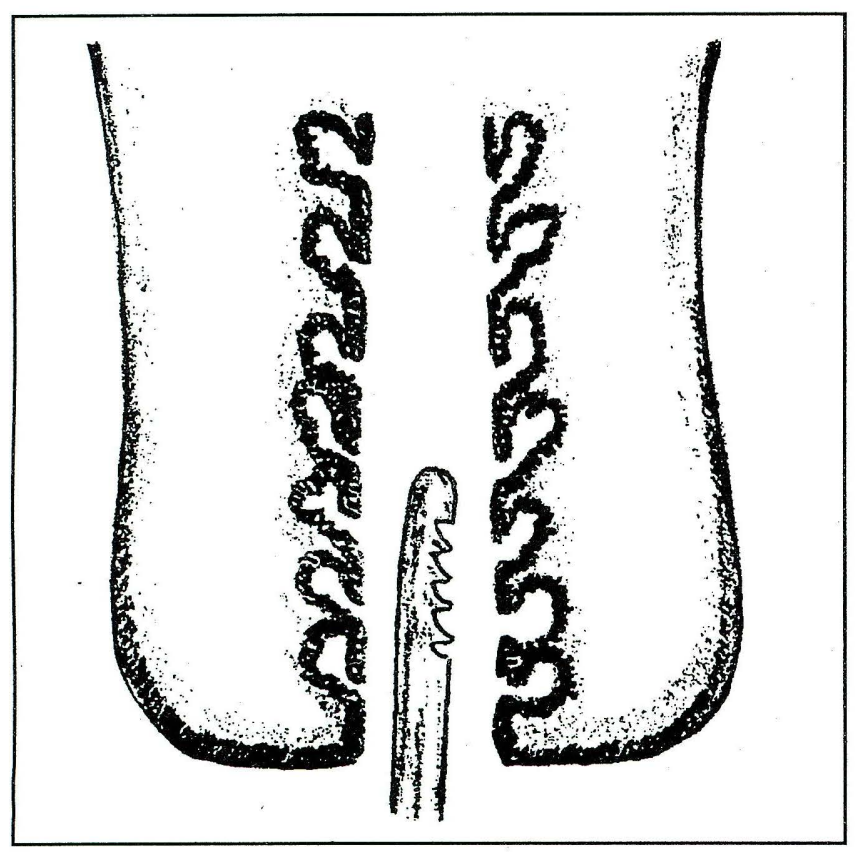

su estudio histopatológico, ya que la exuberancia glandular protruye hacia la luz, facilitando su extracción. Estos son los casos en que es factible clasificar certeramente la severidad de las lesiones y aun definir la presencia de infiltración al estroma. En los casos en que las neoplasias son originadas sobre epitelio metaplásico endocervical, el arrancamiento provocado por la Cureta de Novak soltará las capas superficiales del epitelio escamoso, dificultando la graduación de las lesiones por la ausencia de las capas basales.

En oposición a todas las características negativas del R.E.C., podemos resaltar la calidad del tejido obtenido con radiocirugía que es un procedimiento bien tolerado y sin complicaciones que nos brinda un excelente material para estudio siempre y cuando utilicemos adecuadamente el equipo con el voltaje indicado para evitar quemaduras en los bordes de la pieza y exceso en el sangrado, la sonda indicada y bajo visión directa a través del colposcopio. De esta manera en la gran mayoría de los casos obtenemos una muestra cuboidal que en promedio mide entre 5 y $8 \mathrm{~mm}$ de altura por 4 a $5 \mathrm{~mm}$ de ancho, material este de características óptimas para el estudio histopatológico por su facilidad de orientación de sus extremos distal y proximal, integridad de la arquitectura glandular del canal endocervical con suficiente tejido estromal que permite visualizar claramente la membrana basal. (Lámina 1 y 2)

Para el estudio microscópico se practican cortes longitudinales de la totalidad de la pieza, permitiéndole al patólogo localizar el sitio de la neoplasia, clasificar adecuadamente su severidad y descartar o confirmar inequívocamente la presencia de infiltración al estroma. (Fig. 5).

\section{Conclusiones}

Todas las muestras obtenidas con radiocirugía de las 96 pacientes con sospecha citológica de neoplasia y colposcopia insatisfactoria fueron diagnosticadas positivas para neoplasia, lo que indica una sensibilidad del procedimiento del cien por ciento.

En las pacientes con sospecha citológica de neoplasia y colposcopia insatisfactoria, la confirmación de este diagnóstico por raspado endocervical, fue de solo el $47.27 \%$ que representa una sensibilidad del procedimiento de $47 \%$ por ciento.

En el grupo de pacientes con colposcopia satisfactoria y citología sospechosa de NIC, solo en un $4.8 \%$ se confirmó el diagnóstico y siempre coincidió con el diagnóstico correspondiente a la biopsia exocervical, en ningún caso fue de mayor severidad. La colposcopia bien realizada y satisfactoria es considerada como parámetro seguro para localizar las lesiones cervicales, sin embargo, pueden existir casos muy raros de neoplasia intraepitelial cervical endocervicales con colposcopia totalmente negativa y satisfactoria que pasarían totalmente desapercibidas de no ser sugeridas por un adecuado estudio citológico del endocérvix obtenido con cepillo que justifique la investigación histológica del canal endocervical. Este es el caso de pacientes con citología sospechosa para NIC o sugestiva de adenocarcinoma con estudios colposcópicos negativos y satisfactorios que en el pasado se les indicaba la realización de una conización diagnóstica ante la incapacidad de llegar a un diagnóstico concordante con la citología a pesar de varios raspados endocervicales.

La sensibilidad de la biopsia endocervical cuboidal con radiocirugía para detectar neoplasia endocervical, queda suficientemente documentada y por lo tanto debemos abandonar el raspado endocervical con cureta de Novak rutinizado en la práctica ginecológica.

Cuando consideremos la necesidad de un estudio histológico del canal endocervical, nuestra responsabilidad es obtener el tejido óptimo para tal estudio y no basta con la intención de obtenerlo.

\section{BIBLIOGRAFÍA}

1. Richardt, Wright T. Controversies in the Manegement of low-Grade Cervical IntraEpit Helial Neoplasia: Cancer 1993; 1413-1421.

2. Kossl the Papanicolaou test for cervical cancer detection: a triumph and a tragedy: Jama 1989; 261: 737.

3. Prendiville W. Cullimore J. Normans Large loopexcision transformation zone: a new method of manageueo t. for women with cervical intraepitelial neoplasia Br. journal obstet gynecol 1989, 96: 96-1040-1060.
4. White PF., Olar ks, Treatament of cervical intraepithelial neoplasia: experience with the low-voltage diathermy loop are jogstet gynecol 1990; 162: 1272.

5. Bennett B., Stone Keith Etal. Deep Loop excision for prehisterectomy endocervical evaluation: Am L. Obstet Gynecol 1997; 176: 82-86. 